\title{
ORIGINAL ARTICLE Peripheral nerve alterations after spinal cord injury in the adult rat
}

\author{
E Redondo-Castro and X Navarro
}

Objective: To assess if spinal cord injury ( $\mathrm{SCl}$ ) can produce alterations in axons of peripheral nerves emerging caudal to the injury. Methods: Mild/severe contusion or complete transection was performed at T8 in adult rats. The function and morphology of the sciatic nerve were assessed 3 months after the lesion.

Results: There was a decrease in the amplitudes of muscle responses in nerve conduction tests. The number of myelinated fibers was maintained, but some of them presented structural abnormalities.

Conclusion: SCls cause alterations in peripheral axons not affected by the injury. Preservation of the peripheral components is essential for potential regenerative and rehabilitation therapies. Thus, special care has to be taken to avoid secondary complications, due to compressions or immobility, in $\mathrm{SCl}$ humans.

Spinal Cord (2013) 51, 630-633; doi:10.1038/sc.2013.57; published online 18 June 2013

Keywords: spinal cord injury; axonal count; myelinated nerve fibers; peripheral nerve; sciatic nerve

\section{INTRODUCTION}

Spinal cord injuries (SCIs) cause disruption of ascending and descending pathways, leading to paralysis and loss of sensitivity below the lesion site, as well as positive symptoms such as spasticity and neuropathic pain. It is generally assumed that SCIs do not affect the peripheral systems, but the consequences of an SCI can expand to remote regions, cranially and caudally, and lead to secondary plastic changes involving supraspinal circuits and also the peripheral nervous system. $^{1-3}$ The motoneuron pools distal to the lesion may suffer atrophy and degeneration. ${ }^{4,5}$ The SCI may also affect the central axons of primary sensory neurons conveyed in the dorsal column tracts. Central axotomy and retrograde degeneration may affect the neuron soma in the dorsal root ganglia and lead to degeneration of the peripheral axon branch. ${ }^{4}$ Indeed, changes in peripheral excitability and function have already been described in human SCI patients, ${ }^{2,3,6,7}$ mainly using electrophysiological techniques.

Maintenance of peripheral nerve functions is essential in SCI rehabilitation, regenerative therapies, surgical bridges and neuroprosthetic stimulation strategies. ${ }^{1,3,6-8}$ In this work, we have performed histological and electrophysiological analyses of the peripheral nerve following SCIs of different severities in adult rats, in order to assess if there were secondary peripheral alterations, and if the severity of the injury was correlated with these alterations.

\section{MATERIALS AND METHODS}

Adult female Sprague-Dawley rats ( 8 weeks old; $250-300 \mathrm{~g}$ ) were housed with free access to food and water at a room temperature of $22 \pm 2{ }^{\circ} \mathrm{C}$ under a 12:12 h light-dark cycles. All experimental procedures were approved by the Ethics Committee of our institution, and followed the European Communities Council Directive 86/609/EEC. All applicable institutional and governmental regulations concerning the ethical use of animals were followed during the course of this research. Researchers participating in this work were blinded to the severity of the injury received by animals.

\section{Surgical procedure}

Operations were performed under pentobarbital anesthesia $\left(50 \mathrm{mg} \mathrm{kg}^{-1}\right.$ intraperitoneally, Sigma, St Louis, MO, USA), and after subcutaneous injection of buprenorphine $\left(0.05 \mathrm{mg} \mathrm{kg}^{-1}\right.$, Buprex, Schering-Plough) near the incision site. After dorsal laminectomy of the T8-T9 vertebra, the spinal cord was contused at T8 level using the Infinite Horizon impactor device (Precision Scientific Instruments; Lexington, UK) by applying a force of 100 kilodynes (kdyn; group $100 \mathrm{kdyn}, n=5$ ) or $200 \mathrm{kdyn}$ (group $200 \mathrm{kdyn}, n=5$ ); displacement and force applied were collected for each contusion. In another group of animals, the spinal cord was completely transected (complete section group, $n=5$ ), by means of a sharp scalpel, at T8 vertebral level. To ensure that the injury transected the whole spinal cord, both stumps were gently lifted away and repositioned back into the vertebral channel. In all the surgeries, the wound was sutured with 5/0 silk thread at the muscular plane, and the skin closed with small surgical clips and disinfected with povidone iodine. Animals were kept in a warm environment until full recovery from anesthesia. Bladders were expressed twice a day until reflex voiding of the bladder was reestablished. Amoxicillin was given in the drinking water for 1 week to prevent postoperative infections.

\section{Evaluation of locomotion}

Locomotor hindlimb function and recovery was assessed using the Basso, Beattie and Bresnahan rating scale in open-field walking. ${ }^{9}$ Briefly, the Basso, Beattie and Bresnahan testing scale consists of an ordinal scale from 0 points (no discernable hindlimb movement) to 21 points (consistent, coordinated gait with parallel paw placement of the hindlimb and consistent trunk stability). For measuring locomotor recovery, one animal at a time was allowed to move freely inside a circular plastic tray $(90 \mathrm{~cm}$ diameter $\times 24 \mathrm{~cm}$ wall height) for $5 \mathrm{~min}$, and two examiners observed the hindlimbs' movements of the rat. The

Department of Cell Biology, Physiology and Immunology, Centro de Investigación Biomédica en Red sobre Enfermedades Neurodegenerativas (CIBERNED), Institute of Neurosciences, Universitat Autònoma de Barcelona, Bellaterra, Spain

Correspondence: Dr X Navarro, Unitat de Fisiologia Mèdica, Edif M, Universitat Autònoma de Barcelona, Bellaterra E-08193, Spain.

E-mail: xavier.navarro@uab.cat

Received 9 January 2013; revised 29 April 2013; accepted 14 May 2013; published online 18 June 2013 
final score of each animal was the mean value of both examiners. Locomotion testing was performed weekly until 90 days postoperation.

\section{Peripheral nerve conduction tests}

Electrophysiological tests were performed preoperatively and after 3 months of follow-up. Animals were anesthetized with pentobarbital $\left(30 \mathrm{mg} \mathrm{kg}^{-1}\right.$, intraperitoneally) and placed prone over a warmed flat coil controlled by a hotwater-circulating pump to maintain body temperature. Single electrical pulses ( $100 \mu$ s duration at supramaximal intensity) were delivered by monopolar needles $(27 \mathrm{G})$ inserted percutaneously at the sciatic notch. The compound muscle action potentials (CMAP) were recorded from the anterior tibialis and from the plantar interosseus muscles by means of an active electrode inserted into the belly of the muscle and the reference electrode at the fourth toe. ${ }^{10,11}$ CMAPs were amplified, filtered (bandpass $1 \mathrm{~Hz}-5 \mathrm{kHz}$ ), displayed on an electromyograph (Sapphyre 4ME, Vickers, Surrey, UK) and measured. The responses with the highest amplitude were selected and used for analysis. Values from both hindlimbs of each animal were averaged.

\section{Histological processing and axonal counting}

At 90 days postoperation, the animals were perfused with $4 \%$ paraformaldehyde in $0.1 \mathrm{M}$ phosphate buffer. The sciatic nerve was harvested proximal to its trifurcation (distal part of the thigh), and fixed with Webster's fixative $3 \%$ paraformaldehyde and $3 \%$ glutaraldehyde in $0.1 \mathrm{M}$ phosphate buffer) overnight. The nerve segments were postfixed with $2 \%$ osmium tetroxide in $0.1 \mathrm{M}$ phosphate buffer for $2 \mathrm{~h}$. Samples were dehydrated using increasing graded solutions of ethanol, incubated in propylene oxide for $1 \mathrm{~h}$ and then in a mixture of 50\% propylene oxide and 50\% Epon for 90 min, followed by incubation in 100\% Epon overnight. Transverse sections $0.5 \mu \mathrm{m}$ thick were taken in an ultramicrotome, collected into glass slides, and stained with toluidine blue. Images were acquired at $\times 100$ under light microscope and counts of the myelinated fibers were performed using $\mathrm{NIH}$ Image J software (Bethesda, MD, USA). The density of myelinated fibers was obtained from counts in fields chosen by systematic sampling, representing at least $50 \%$ of the total area of the nerve. The total number of myelinated fibers was calculated by multiplying the myelinated fiber density per cross-sectional area of the nerve. ${ }^{12,13}$

\section{RESULTS}

Locomotion

After the SCI, the rats showed a reduction in the Basso, Beattie and Bresnahan score in relation with the severity of the lesion (Figure 1a). Animals receiving $100-\mathrm{kdyn}$ contusion reached a plateau at $15-16$ points, indicating plantar stepping and coordinated gait, although with some deficits regarding toe clearance and rotation of the hindpaw position; rats of the 200-kdyn contusion group presented more marked deficits, such as uncoordinated gait and only occasional plantar stepping (score about 9-10 points). Animals with complete transection displayed nearly complete paralysis, therefore, they reached scores of 1-2 points during the follow-up (only slight movement of some joints). There were significant differences between the three groups $(P<0.001)$ at all time points after injury.

\section{Electrophysiology}

CMAPs of the anterior tibialis muscle showed a progressive reduction as the severity of the injury increased (Figure 1b). Intact animals had a mean amplitude of $52.3 \pm 1.4 \mathrm{mV}$, whereas animals with mild and severe contusions presented slightly reduced values (100 kdyn: $47.4 \pm 1.8 \mathrm{mV}$; $200 \mathrm{kdyn}: 47.8 \pm 1.6 \mathrm{mV}$ ), and complete section animals showed a significant reduction $(40.8 \pm 1.5 \mathrm{mV}, P<0.05$ versus presurgical values). The latency presented a nonsignificant reduction in all injured groups (intact latency: $\sim 1.45 \mathrm{~ms}$; injured animals $\sim 1.30 \mathrm{~ms}$ ). Latencies and amplitudes of the plantar muscles' CMAPs a Open field locomotion
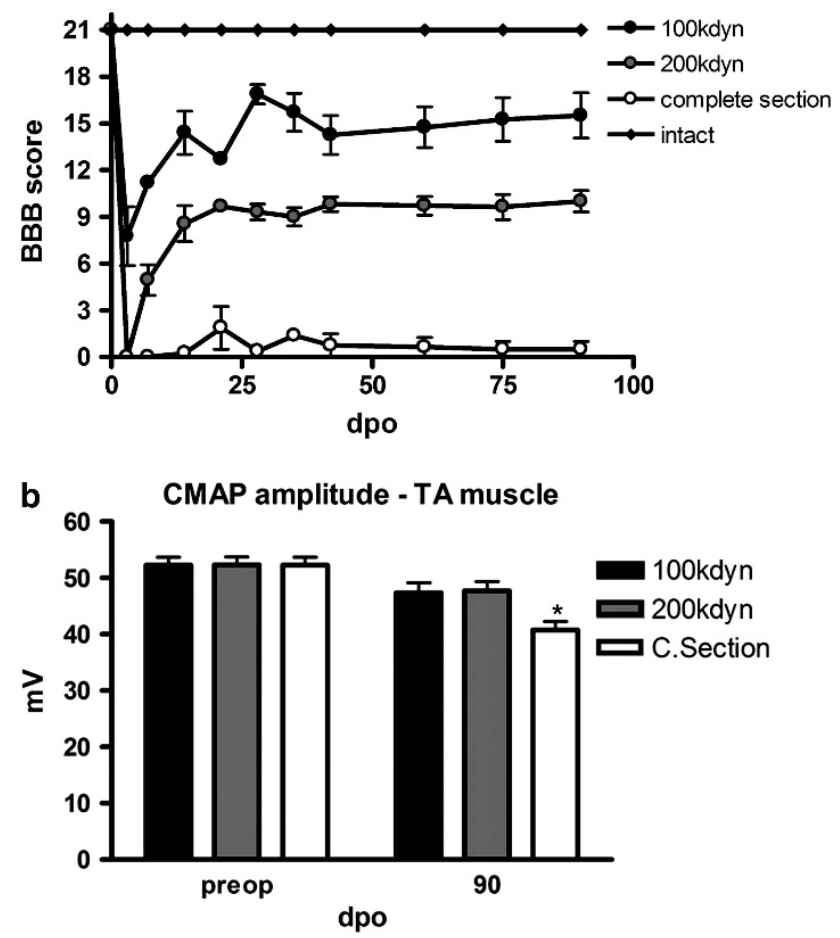

Figure 1 (a) Locomotor evaluation (Basso, Beattie and Bresnahan, BBB score) indicates graded functional deficits related to the severity of the injury. Groups displayed results significantly different between them at all time points $(P<0.05)$. (b) Amplitude of the CMAP recorded in the tibialis anterior muscle (TA) 90 days postoperation (dpo). There was a reduction in amplitude after $\mathrm{SCl}$ compared with prelesional values, which was only significant in the complete section group $\left({ }^{*} P<0.05\right.$ versus prelesional value).

displayed similar results, with a small reduction in amplitude and no changes in latencies.

\section{Histology}

Axonal counts were done to assess if the reduction of CMAP amplitude was due to axonal loss. Measurements were performed in the tibial nerve, the main fascicle of the sciatic nerve. Injured rats had a total number of myelinated fibers similar to intact nerves (Figure 2a; intact: $4851 \pm 143$; $100 \mathrm{kdyn}: 4864 \pm 129 ; 200 \mathrm{kdyn}$ : $4629 \pm 29$; section: $4650 \pm 120$ ), but they had an increased number of fibers with abnormal appearance (Figure 2b; intact: $99 \pm 21$; 100 kdyn: $277 \pm 46$; $200 \mathrm{kdyn}: 262 \pm 51$; section: $438 \pm 45$ ). The abnormalities observed were mainly detachment of the myelin sheath and axonal atrophy, but no clear signs of degeneration were found in any of the rats (Figure 2c). Some of these altered figures correspond to SchmidtLanterman incisures. The abnormal fibers did not display a fascicular organization within the section, although in SCI groups they were more frequent at the periphery of the tibial nerve cross-section. The sural and peroneal fascicles of the sciatic nerve did not display such noticeable abnormalities.

\section{DISCUSSION}

The effects of an SCI are not restricted to the spinal cord itself, but can expand to remote areas of the spinal cord as well as to the peripheral nervous system. ${ }^{1,3}$ Our results indicate that thoracic SCI causes clear functional deficits, especially visible in locomotion. These 




b



C



d

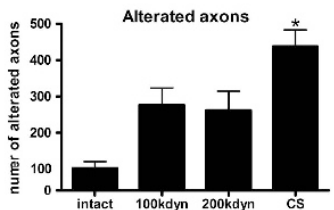

Figure 2 (a) Microphotographs of the periphery of the tibial fascicle of the sciatic nerve in different experimental groups (scale bar $100 \mu \mathrm{m}$ ). Right panels show details of the altered fibers, indicated by asterisks (scale bar $10 \mu \mathrm{m}$ ). (b) Image at low magnification of the sciatic nerve with the three main fascicles. The dark area indicates the periphery of the fascicle, where most abnormal axons tend to be placed (scale bar $0.5 \mathrm{~mm}$ ). (c) The total number of myelinated axons is not significantly changed in any of the groups when compared with intact animals, but the number of abnormal axons (d) tends to increase with the severity of the injury $\left({ }^{*} P<0.05\right.$ versus intact rats).

deficits are directly related to the severity of the injury received. ${ }^{14-16}$ On the other hand, SCI did not cause axonal degeneration in the sciatic nerve, although it induced some axonal alterations, despite the lesion did not primarily affect its contributing neurons. Electrophysiological tests showed also a mild functional decline of the CMAP amplitude in muscles of the hindlimb.

Three months after a thoracic SCI, there was no significant loss of myelinated axons in the sciatic nerve compared with intact nerves. However, the number of abnormalities observed in myelinated fibers increased, particularly in the rats with complete cord section. We cannot ensure that these alterations would eventually lead to degeneration, as there were not typical features of axonal degeneration (disorganized myelin-forming bundles, presence of myelin bodies in the axoplasm, swelling, hyperplasia, axonal atrophy and so on). Indeed, the observed alterations may correspond to SchmidtLanterman incisures, placed near the nodes of Ranvier, where the myelin sheath is partially reorganized. The increased presence of these cisures in the injured animals may be considered as a sign of nerve fiber dysfunction. In fact, an increase of these structures was described at the onset of Wallerian degeneration, ${ }^{17,18}$ and in nerve compression injuries. ${ }^{19}$ As we found these abnormal figures predominantly at the periphery of the nerve, we may consider the compression hypothesis as one of the most feasible explanations. Regarding the type of altered axons, our observations are suggestive that motor axons are the ones more likely to be affected, as abnormalities were predominantly located at the periphery of the tibial nerve, where motor axons cluster at higher density. ${ }^{20}$

The loss of function of the hindlimbs after the SCI causes muscle atrophy, but immobilization and muscle atrophy by disuse seem to have small effects on the decline of CMAP amplitudes and histological outcomes, ${ }^{2,21}$ as our results indicate. Conversely, axonal degeneration has been reported in humans after SCI. This leads to the assumption that the loss detected in humans may be secondary not only to disuse, but also to compression in paralyzed limbs. Other causes may include edema caused by inactivity and reduced blood flow following SCI. ${ }^{1,2}$ As locomotion is more easily recovered in rodents than in humans after SCI, the secondary complications associated to disuse and immobility would be less important in quadrupedal animals. As an example, paraplegic humans maintain their legs immobilized for long time periods, whereas paraplegic rodents may move them passively while walking with forelimbs. Prolonged immobilization, in addition to the muscle loss, can lead to nerve compressions that may explain some alterations in peripheral nerves, which would be more marked in humans than in rodents. Special attention must be given to avoid such secondary complications of SCI in humans (disuse, immobility, atrophy, nerve compressions) that may eventually lead to peripheral nerve damage and loss of functionality. This should be taken into consideration when designing rehabilitation or regenerative therapies, providing special care to maintain peripheral neuromuscular function. ${ }^{1,6,7}$

\section{CONFLICT OF INTEREST}

The authors declare no conflict of interest.

\section{ACKNOWLEDGEMENTS}

This work was supported by grants from the Fundació MaratóTV3 (grant 070210) and the Ministerio de Ciencia y Innovación (grant SAF2009-12495), and funds from Red de Terapia Celular (TERCEL) of Spain and FEDER. We 
thank the technical assistance of Monica Espejo and Marta Morell, the surgical help of Abel Torres-Espín and the histological work of Jessica Jaramillo.

1 Riley DA, Burns AS, Carrion-Jones M, Dillingham TR. Electrophysiological dysfunction in the peripheral nervous system following spinal cord injury. PMR 2011; 3: 419-425; quiz 425.

2 Van De Meent H, Hosman AJ, Hendriks J, Zwarts MEM-SCI Study GroupSchubert M. Severe degeneration of peripheral motor axons after spinal cord injury: a European multicenter study in 345 patients. Neurorehabil Neural Repair 2010; 24: 657-665.

3 Lin CS-Y, Macefield VG, Elam M, Wallin BG, Engel S, Kiernan MC. Axonal changes in spinal cord injured patients distal to the site of injury. Brain 2007; 130: 985-994.

4 Kitzman P. Alteration in axial motoneuronal morphology in the spinal cord injured spastic rat. Exp Neurol 2005; 192: 100-108.

5 García-Alías G, Torres-Espín A, Vallejo C, Navarro X. Functional involvement of the lumbar spinal cord after contusion to T8 spinal segment of the rat. Restor Neurol Neurosci 2010; 28: 781-792.

6 Berman SA, Young RR, Sarkarati M, Sherfner J. Injury zone denervation in traumatic quadriplegia in humans. Muscle Nerve 1996; 19: 701-706.

7 Nogajski J, Engel S, Kiernan MC. Focal and generalized peripheral nerve dysfunction in spinal cord-injured patients. J Clin Neurophysiol 2006; 23: 273-279.

8 Mackinnon SE, Yee A, Ray WZ. Nerve transfers for the restoration of hand function after spinal cord injury. J Neurosurg 2012; 117: 176-185.

9 Basso DM, Beattie MS, Bresnahan JC. A sensitive and reliable locomotor rating scale for open field testing in rats. J Neurotrauma 1995; 12: 1-21.

10 Valero-Cabré A, Navarro X. H reflex restitution and facilitation after different types of peripheral nerve injury and repair. Brain Res 2001; 919: 302-312.
11 Valero-Cabré A, Forés J, Navarro X. Reorganization of reflex responses mediated by different afferent sensory fibers after spinal cord transection. J Neurophysiol 2004; 91 : 2838-2848.

12 Bruna J, Alé A, Velasco R, Jaramillo J, Navarro X, Udina E. Evaluation of pre-existing neuropathy and bortezomib retreatment as risk factors to develop severe neuropathy in a mouse model. J Peripher Nerv Syst 2011; 16: 199-212.

13 Gómez N, Cuadras J, Buti M, Navarro X. Histologic assessment of sciatic nerve regeneration following resection and graft or tube repair in the mouse. Restor Neurol Neurosci 1996; 10: 187-196.

14 Redondo-Castro E, Udina E, Verdú E, Navarro X. Longitudinal study of wind-up responses after graded spinal cord injuries in the adult rat. Restor Neurol Neurosci 2011; 29: 115-126.

15 Scheff SW, Rabchevsky AG, Fugaccia I, Main JA, Lumpp JE Jr. Experimental modeling of spinal cord injury: characterization of a force-defined injury device. J Neurotrauma 2003; 20: 179-193.

16 Cao Q, Zhang YP, lannotti C, DeVries WH, Xu XM, Shields CB et al. Functional and electrophysiological changes after graded traumatic spinal cord injury in adult rat. Exp/ Neurol 2005; 191 (Suppl), S3-S16.

17 Webster $H$. The relationship between Schmidt-Lanterman incisures and myelin segmentation during Wallerian degeneration. Ann N Y Acad Sci 1965; 31: 29-38.

18 Hall SM, Williams PL. Studies on the 'incisures' of Schmidt and Lanterman. J Cell Sci 1970; 6: 767-791.

19 Hunter DA, Moradzadeh A, Whitlock EL, Brenner MJ, Myckatyn TM, Wei CH et al. Binary imaging analysis for comprehensive quantitative histomorphometry of peripheral nerve. J Neurosci Methods 2007; 166: 116-124.

20 Badia J, Pascual-Font A, Vivó M, Udina E, Navarro X. Topographical distribution of motor fascicles in the sciatic-tibial nerve of the rat. Muscle Nerve 2010; 42: 192-201.

21 Urso ML, Clarkson PM, Price TB. Immobilization effects in young and older adults. Eur J Appl Phys 2006; 96: 564-571. 